\title{
QUEEN'S
UNIVERSITY
BELFAST
}

\section{Extracting bridge frequencies from dynamic responses of two passing vehicles}

Kim, C. W., Inoue, S., Sugiura, K., McGetrick, P., \& Kawatani, M. (2016). Extracting bridge frequencies from dynamic responses of two passing vehicles. In A. Zingoni (Ed.), Insights and Innovations in Structural Engineering, Mechanics and Computation: Proceedings of the Sixth International Conference on Structural Engineering, Mechanics and Computation, Cape Town, South Africa, 5-7 September 2016 (pp. 1858-1864). CRC Press. https://www.crcpress.com/Insights-and-Innovations-in-Structural-Engineering-Mechanics-andComputation/Zingoni/p/book/9781138029279

Published in:

Insights and Innovations in Structural Engineering, Mechanics and Computation: Proceedings of the Sixth International Conference on Structural Engineering, Mechanics and Computation, Cape Town, South Africa, 5-7 September 2016

Document Version:

Peer reviewed version

Queen's University Belfast - Research Portal:

Link to publication record in Queen's University Belfast Research Portal

\author{
Publisher rights \\ (C) 2016 CRC Press, Taylor \& Francis Group \\ This is an Accepted Manuscript of a paper published by CRC Press in Insights and Innovations in Structural Engineering, Mechanics and \\ Computation on August 15, 2016, available online: https://www.crcpress.com/Insights-and-Innovations-in-Structural-Engineering-Mechanics- \\ and-Computation/Zingoni/p/book/9781138029279
}

\section{General rights}

Copyright for the publications made accessible via the Queen's University Belfast Research Portal is retained by the author(s) and / or other copyright owners and it is a condition of accessing these publications that users recognise and abide by the legal requirements associated with these rights.

Take down policy

The Research Portal is Queen's institutional repository that provides access to Queen's research output. Every effort has been made to ensure that content in the Research Portal does not infringe any person's rights, or applicable UK laws. If you discover content in the Research Portal that you believe breaches copyright or violates any law, please contact openaccess@qub.ac.uk. 


\title{
Extracting bridge frequencies from dynamic responses of two passing vehicles
}

\author{
C.W. Kim, S. Inoue \& K. Sugiura \\ Dept. of Civil and Earth Resources Eng., Graduate School of Eng., Kyoto University, Kyoto 615-8540, Japan \\ P.J. McGetrick \\ SPACE, Queen's University Belfast BT9 5AG, UK
}

M. Kawatani

Dept. of Civil Eng., Graduate School of Eng., Kobe University, Kobe 657-8501, Japan

\begin{abstract}
A conventional way to identify bridge frequencies is utilizing vibration data measured directly from the bridge. A drawback with this approach is that the deployment and maintenance of the vibration sensors are generally costly and time-consuming. One of the solutions is in a drive-by approach utilizing vehicle vibrations while the vehicle passes over the bridge. In this approach, however, the vehicle vibration includes the effect of road surface roughness, which makes it difficult to extract the bridge modal properties. This study aims to examine subtracting signals of two trailers towed by a vehicle to reduce the effect of road surface roughness. A simplified vehicle-bridge interaction model is used in the numerical simulation; the vehicle - trailer and bridge system are modeled as a coupled model. In addition, a laboratory experiment is carried out to verify results of the simulation and examine feasibility of the damage detection by the drive-by method.
\end{abstract}

\section{INTRODUCTION}

Monitoring aged and deteriorated structures including bridges has been an important technical issue in many countries. For bridge structures, structural health monitoring based on vibration data has become a more popular tool for the condition assessment of bridges. A conventional way to identify bridge frequencies is utilizing vibration data measured directly from the bridge, using vibration sensors mounted on the bridge. A drawback with this approach is that the deployment and maintenance of the vibration sensors are generally costly and timeconsuming. One of the solutions is in a drive-by approach (e.g. Yang et al. 2004, Kim et al. 2014).

The feasibility of extracting natural frequencies of bridges from vehicle vibrations has been verified theoretically. The practical feasibility of this method was examined experimentally by passing an instrumented vehicle over a highway bridge in Taiwan (Lin et al. 2005). Toshinami et al. (2010) and Kim et al. (2014) presented the results of scaled laboratory experiments aiming at verifying the feasibility of a dive-by inspection approach and concluded the importance of removing roadway surface roughness to improve identifiability. Two approaches can be considered to increase the possibility of successful identification. One approach is to increase the vibration amplitude of the bridge by allowing the bridge to be exposed to existing traffic or accompanying vehi- cles. The other approach is to reduce or eliminate the effect of roadway surface roughness. To eliminate or reduce the effect of road surface roughness by first synchronizing the response of the two vehicles with respect to the same contact points, and then by subtracting the synchronized response of one vehicle from the other.

In this study, a theoretical derivation was first presented to clarify the mechanisms of the drive-by inspection approach using two passing vehicles. Then a numerical simulation and a laboratory experiment were carried out to investigate the feasibility of this approach. A simplified vehicle-bridge interaction model was used in the numerical simulation; the vehicle-trailer and bridge system were modeled as a coupled model that allows multiple vehicles crossing the bridge. In addition, the laboratory experiment was carried out to examine of its application to damage detection as well.

\section{THEORETICAL STUDY}

The vehicle response contains not only dynamic characteristics of the bridge and vehicle but also other information such as the driving-related frequencies and road surface effects, which makes the extraction of bridge frequencies difficult. In order to investigate the feasibility of a drive-by inspection approach theoretically, two cases both a single vehicle and two vehicles are adopted (e.g. Yang et al. 2012). 


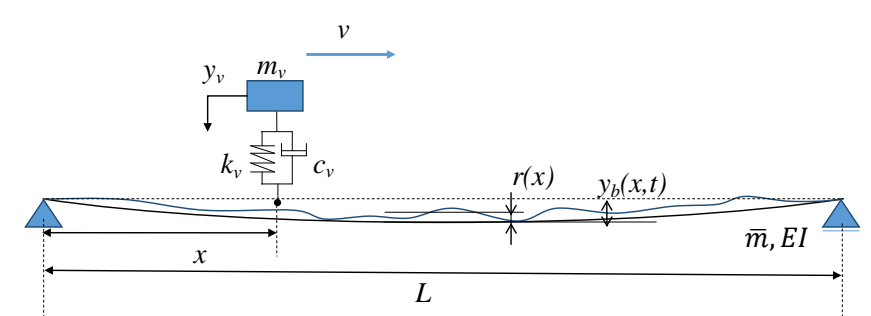

Figure 1. Model of a single vehicle passing through a simple bridge

\subsection{Theoretical study of a single vehicle traveling on a simple bridge}

Figure 1 shows the two-dimensional vehicle-bridge interaction model, where the vehicle is modeled as a moving sprung mass $m_{v}$ supported by a spring of stiffness $k_{v}$ and the simple bridge is modeled as a Bernoulli-Euler beam of length $L$, mass density $\bar{m}$ per unit length and bending rigidity EI. The road surface roughness profile is denoted by $r(x)$, a function of $x$ in the longitudinal direction of the bridge.

Let the vehicle move over the bridge with speed $v$. The equation of motion for both the vehicle and bridge at time $t$ can be expressed as follows by neglecting the damping effect of the vehicle and bridge for the simplicity.

$$
\begin{aligned}
& m_{v} \ddot{y}_{v}(t)+\left.k_{v}\left[y_{v}(t)-y_{b}(x, t)-r(x)\right]\right|_{x=v t}=0 \\
& \bar{m} \ddot{y}_{b}(x, t)+E I y_{b} " '(x, t)=f_{c}(t) \delta(x-v t)
\end{aligned}
$$

where $y_{v}$ and $y_{b}$ denote the vertical displacement of the vehicle and bridge respectively, and the contact force $f_{c}$ is defined as follows.

$f_{c}(t)=-m_{v} g+\left.k_{v}\left[y_{v}(t)-y_{b}(x, t)-r(x)\right]\right|_{x=v t}$

The road surface roughness is defined as follows.

$r(x)=\sum_{i} d_{i} \cos \left(n_{i} x+\theta_{i}\right)$

where $n_{i}$ is the $i$ th spatial frequency and $d_{i}$ and $\theta_{i}$ are the amplitude and the random phase angle of $i$ th cosine function respectively.

By the modal superposition method, the bridge response can be expressed in terms of the mode vectors and generalized coordinates. The solution to Equation 2 can be expressed as follows.

$$
y_{b}(x, t)=\sum_{n=1}^{\infty} \sin \frac{n \pi x}{L} q_{n}(t)
$$

Substituting Equation 5 into Equation 2 and integrating the variable $x$ from 0 to $L$, Equation 6 can be obtained.

$$
\begin{aligned}
\ddot{q_{n}}+\omega_{b, n}^{2} q_{n}= & 2 \mu \sin n l(t)\{-g \\
& +\omega_{v}^{2}\left[y_{v}(t)-y_{b}(x, t)\right. \\
& -r(x)]\left.\right|_{x=v t}
\end{aligned}
$$

where $l(t)=\pi v t / L, \mu=m_{v} / \bar{m} L$ and $\omega_{b, n}$ is the angular frequency of the bridge for the $n$th mode and $\omega_{v}$ is the angular frequency of the vehicle, which are defined as Equation 7. $\omega_{b, n}=\frac{n^{2} \pi^{2}}{L^{2}} \sqrt{\frac{E I}{\bar{m}}}, \quad \omega_{v}=\sqrt{\frac{k_{v}}{m_{v}}}$

On the right side of Equation 6, the second and last terms are the elastic force of the suspension and the inertial force of the vehicle caused by the surface roughness respectively. These two terms have negligible effect on the response of the bridge for simplicity as they usually cause less than $5 \%$ difference in maximum bridge displacement responses (Yau et al. 1999). The equation of motion for the beam in Equation 6 can be simplified as Equation 8.

$$
\ddot{q_{n}}+\omega_{b, n}^{2} q_{n}=2 \sin n l(t)\{-\mu g\}
$$

One can solve Equation 8 to obtain the generalized coordinate $q_{n}$ of the $n$th mode as follows.

$$
q_{n}=\frac{\Delta_{s t, n}}{1-S_{n}^{2}}\left[\sin n l(t)-S_{n} \sin \omega_{b, n} t\right]
$$

where $\Delta_{s t, n}$ is the static deflection caused by the weight of the vehicle as shown in Equation 10.

$$
\Delta_{s t, n}=\frac{-2 m_{v} g L^{3}}{n^{4} \pi^{4} E I}
$$

and $S_{n}$ is a non-dimensional speed parameter defined as follows.

$$
S_{n}=\frac{n \pi v}{L \omega_{b, n}}
$$

Substituting Equation 9 into Equation 5 yields the general solution of the vertical displacement of the bridge as shown in Equation 12.

$$
\begin{gathered}
y_{b}(x, t)=\sum_{n} \frac{\Delta_{s t, n}}{1-S_{n}^{2}}\left\{\sin \frac{n \pi x}{L}[\sin n l(t)\right. \\
\left.\left.-S_{n} \sin \omega_{b, n} t\right]\right\}
\end{gathered}
$$

Substituting Equation 4 and Equation 12 into Equation 1, and applying Duhamel's integral, the vehicle displacement can be obtained. Differentiating it with respect to the time $t$ twice, the vehicle acceleration response can be obtained as follows.

$$
\begin{aligned}
& \ddot{y}_{v}(t) \\
& =\sum_{n=1}^{\infty}\left\{A_{1, n} \cos ((n-1) l(t))\right. \\
& -A_{2, n} \cos ((n+1) l(t))+A_{3, n} \cos \omega_{v} t \\
& \left.-A_{4, n} \cos \left(\omega_{n} t-n l(t)\right)+A_{5, n} \cos \left(\omega_{n} t+n l(t)\right)\right\} \\
& +\sum_{i=1}^{\infty} d_{i} \frac{\omega_{v}^{2}}{\omega_{v}^{2}-\left(n_{i} v\right)^{2}}\left\{-\left(n_{i} v\right)^{2} \cos \left(n_{i} v t+\theta_{i}\right)\right. \\
& \left.+\omega_{v}^{2} \cos \theta_{i} \cos \omega_{v} t+n_{i} v \omega_{v} \sin \theta_{i} \sin \omega_{v} t\right\}
\end{aligned}
$$

Herein the details of coefficients $A_{1, n}, A_{2, n}, A_{3, n}, A_{4, n}$ and $A_{5, n}$ are omitted but available in Yang et al. (2012). 
Table 1 Bridge properties.

\begin{tabular}{lll}
\hline Span length (m) & & 5.4 \\
$1^{\text {st }}$ natural frequency (Hz) & Simulation (Sim) & 3.47 \\
& Experiment (Exp) & 3.64 \\
Damping ratio (Exp) & & 0.011 \\
\hline
\end{tabular}

Table 2 Vehicle properties.

\begin{tabular}{cccccc}
\hline & \multicolumn{2}{c}{ Tractor } & \multicolumn{2}{c}{ Trailer 1 \& 2 } \\
& Sim & Exp & Sim & Exp \\
\hline \multicolumn{2}{c}{ Total mass (kg) } & 19.67 & 19.67 & 7.68 & 7.68 \\
Natural fre- & Front axle & 2.91 & 2.91 & 2.33 & 2.43 \\
quency $(\mathrm{Hz})$ & Rear axle & 2.91 & 2.91 & 2.33 & 2.43 \\
\hline
\end{tabular}

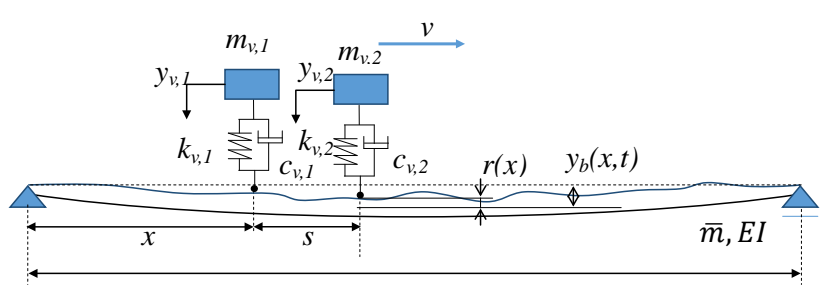

Figure 2. Model of two vehicles passing through a simple bridge.

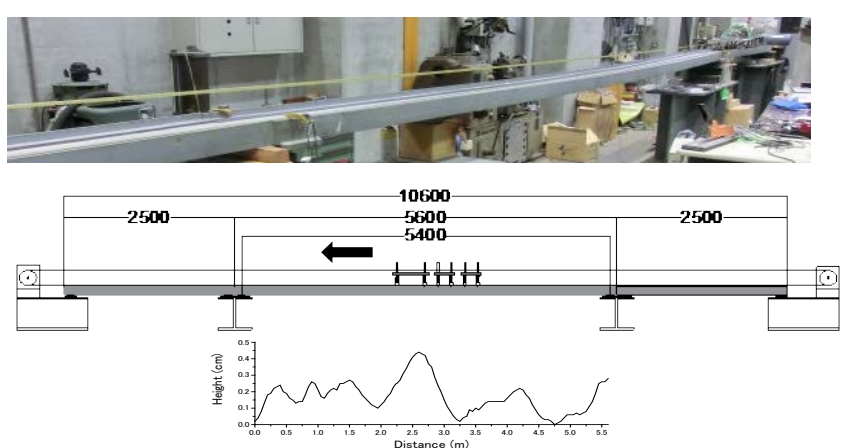

Figure 3. Scaled bridge model and surface roughness profile used in the laboratory experiment.

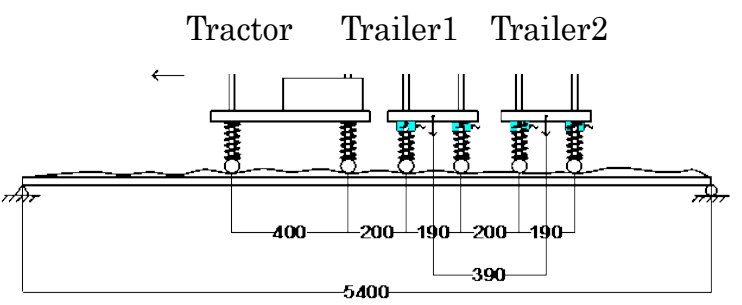

Figure 4. Tractor and trailer models used in the laboratory experiment.

From Equation 13, it is shown that the vehicle response is dominated by four specific groups of frequency: the angular vehicle frequency $\omega_{v}$ (or $f_{v}$ for frequency), angular driving frequency of the moving vehicle $2 \pi v / L$ (or $v / L$ for frequency), shifted angular frequencies of the bridge $\omega_{b} \pm \pi v / L$ (or $f_{b} \pm v /(2 L)$ for frequency), and roughness related frequency $n_{i} v$.

\subsection{Theoretical study of two vehicles traveling on a simple bridge}

As shown in Figure 2, two connected vehicles with fixed spacing s pass through a simple bridge at constant speed $v$ are modeled. The equation of motion for two vehicles and the bridge are

$m_{v} \ddot{y}_{v k}(t)+k_{v}\left(y_{v k}(t)-y_{b}\left(x_{k}, t\right)-r\left(x_{k}\right)\right)=0$

where $k$ denotes the $k$ th vehicle, i.e. $k=1$ and $k=2$ indicate rear and front trailers respectively. $m_{b} \ddot{y}_{b}+E I y_{b}=\sum_{k=1}^{2} f_{c, k}(t) \delta\left(x-x_{k}\right)$

where $f_{c, k}(t)$ is the contact forces for the axle of the $k$ th vehicle.

$$
f_{c, k}(t)=-m_{v} g+k_{v}\left(y_{v k}(t)-y_{b}\left(x_{k}, t\right)-r\left(x_{k}\right)\right)
$$

The displacement of the rear trailer can be solved. Herein, only the part of the response induced by road surface roughness is of our concern, which can be given as follows.

$$
\begin{aligned}
\ddot{y}_{v 1, r}(t)=\sum_{i=1}^{\infty} & d_{i} B_{1}\left\{-\left(n_{i} v\right)^{2} \cos \left(n_{i} v t+\theta_{i}\right)\right. \\
& +\omega_{v, 1}^{2} \cos \theta_{i} \cos \omega_{v, 1} t \\
& \left.+n_{i} v \omega_{v, 1} \sin \theta_{i} \sin \omega_{v, 1} t\right\}
\end{aligned}
$$

where $B_{1}=\omega_{v, 1}^{2} /\left\{\omega_{v, 1}^{2}-\left(n_{i} v\right)^{2}\right\}$.

Similar to Equation 12, $\ddot{y}_{v 1, r}$ is governed by the roughness frequency $n_{i} v$ and angular vehicle frequency $\omega_{v, 1}$. Let us denote the component related to the roughness frequencies as

$$
\begin{aligned}
& \ddot{R}_{v 1, r}(t)=\sum_{i=1}^{\infty} d_{i} B_{1}\left\{-\left(n_{i} v\right)^{2} \cos \left(n_{i} v t+\theta_{i}\right)\right\} \\
& \ddot{R}_{v 2, r}(t)=\sum_{i=1}^{\infty} d_{i} B_{2}\left\{-\left(n_{i} v\right)^{2} \cos \left(n_{i} v t+\theta_{a}\right)\right\}
\end{aligned}
$$

where $B_{k}=\omega_{v, k}^{2} /\left\{\omega_{v, k}^{2}-\left(n_{i} v\right)^{2}\right\}$ in which $k=1$ and $k=2$, the phase angle is defined as $\theta_{a}=n_{i} s+\theta_{i}$, and $s$ denotes the spacing between two vehicles.

As a result, by first synchronizing the accelerations of two vehicles for the same contact point, and then subtracting the synchronized responses from each other, it can be possible to eliminate the effect of road surface roughness.

\section{ANALYTICAL AND EXPERIMENTAL INVESTIGATION}

A vehicle-bridge interaction (VBI) was simulated utilizing a 2 degree-of-freedom (DOF) half-car model crossing over a finite element (FE) beam at constant speed. Methodology has been available in detail by McGetrick et al. (2009).

A scaled moving vehicle laboratory experiment was performed to investigate the feasibility of the drive-by approach. The experiment setup and roadway profiles considered in the experiment are shown in Figure 3. Three simple beams are used in the experiment in which each span is for accelerating, decelerating and observation respectively. Roadway profiles were considered in the experiment. The scaled bridge model used in the experiment was a simply supported steel-beam with the span length of $5.4 \mathrm{~m}$. Accelerometers were deployed at quarter, mid- and three-quarter spans to monitor bridge responses in free vibration tests and during crossings 
of the vehicle over the bridge. Physical properties of the beam obtained from the manufacturer and free vibration tests are given in Table 1.

The vehicle model used for measurement comprises three cars consisting of a tractor and two following trailers as shown in Figure 4. The tractor was a two-axle vehicle, which serves to excite the bridge into motion and thus plays the role of an exciter to the bridge. It carries a wireless router and data logger that allows the acceleration data to be recorded remotely. The trailer was a two-axle vehicle, which will be excited by the bridge that is already in vibration and thus serves as a receiver of the bridge motions. Two accelerometers were mounted at the centre of the front and rear axles respectively to monitor the trailer bounce motion. The two trailers were assembled with identical components and configurations so as to behave with identical dynamic characteristics. Physical properties of the tractor and trailers models are given in Table 2. The simulated and experimental driving frequency of the moving vehicle $(v / L)$ and two shifted frequencies of the bridge $\left(f_{b} \pm v /(2 L)\right)$ are summarized in Table 3 . The sampling frequency was $100 \mathrm{~Hz}$, and the vehicle speed was $1.05 \mathrm{~m} / \mathrm{s}$.

\section{EXTRACTING BRIDGE FREQUENCY}

\subsection{Numerical simulation}

As mentioned in the previous section, a simplified vehicle-bridge interaction model is used in the numerical simulation to verify validity of extracting bridge frequencies from trailer vibrations. Fourier spectra of Acc1 and Acc2 are shown in Figures 5(a) and (b) in which "Acc1" denotes the acceleration response of Trailer 1 and "Acc2" denotes the acceleration response of Trailer 2. Figure 5(c) shows the Fourier spectra of the residual response, namely FFT[Acc1-Acc2], in which "Acc1-Acc2" indicates the residual acceleration response obtained by subtracting Acc2 from Acc1. Figure 5(d) shows the residual spectra obtained by subtracting one spectrum from the other one, namely FFT[Acc1]-FFT[Acc2]. In addition, vertical red line and blue lines in Figure 5 indicate the frequency of the trailer and the shifted frequencies of the bridge respectively. Moreover FFT[.] denotes the fast Fourier transform of a signal.

In Figures 5(a) and 5(b) the peaks around $2.33 \mathrm{~Hz}$ which is relevant to the trailer frequency dominate. It is noteworthy the dominant spatial frequencies of the roadway surface model $\left(n_{i}\right)$ ranged between $0.3 \mathrm{c} / \mathrm{m}$ and $2.0 \mathrm{c} / \mathrm{m}$, and the peaks at lower frequencies than $2 \mathrm{~Hz}$ are related to the frequency of road surface roughness $\left(n_{i} v\right)$. On the other hand, in Figures 5(c) and 5(d), those peaks in the frequency lower than $2 \mathrm{~Hz}$ as well as that relevant to the trailer frequency were reduced, which indicates that the roughness-related components were reduced by taking FFT[Acc1-Acc2] and FFT[Acc1]-FFT[Acc2]. In addition, the peaks near the shifted bridge frequency were noticeable. However, it is still difficult to identify the bridge frequency clearly. The peaks close to the bridge frequency $(3.47 \mathrm{~Hz})$ in Figure $5(\mathrm{c})$ are larger than those in Figure 5(d), which demonstrates that the frequencies relevant to the road surface roughness and the vehicle were more effectively reduced by FFT[Acc1-Acc2] than FFT[Acc1]FFT[Acc2].

\subsection{Laboratory experiment}

Fourier spectra from the laboratory experiment are shown in Figure 6. In Figures 6(a) and 6(b), major peaks appear at the roughness related frequencies under $2 \mathrm{~Hz}$ and the trailer frequency $(2.43 \mathrm{~Hz})$. Peaks relevant to shifted bridge frequencies were also observed.

Table 3 Shifted bridge frequencies.

\begin{tabular}{ccc}
\hline & Simulation & Experiment \\
\hline$v / L$ & $0.19 \mathrm{~Hz}$ & $0.19 \mathrm{~Hz}$ \\
$f_{b}-v /(2 L)$ & $3.38 \mathrm{~Hz}$ & $3.55 \mathrm{~Hz}$ \\
$f_{b}+v /(2 L)$ & $3.57 \mathrm{~Hz}$ & $3.74 \mathrm{~Hz}$ \\
\hline
\end{tabular}

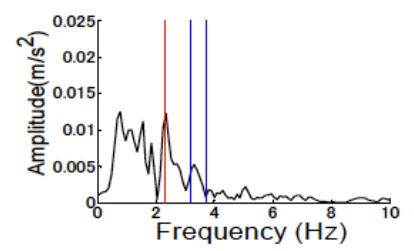

(a) FFT[Acc1]

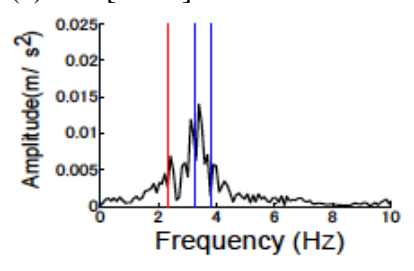

(c) FFT[Acc1-Acc2]

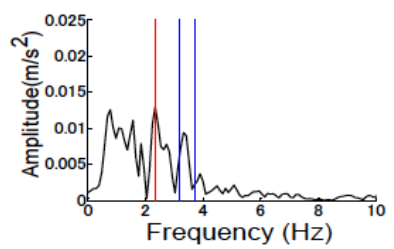

(b) FFT[Acc2]

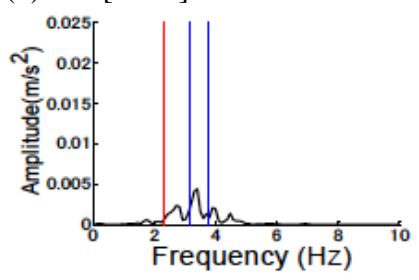

(d) FFT[Acc1]-FFT[Acc2]
Figure 5. Fourier spectra of trailer vibrations from simulation.

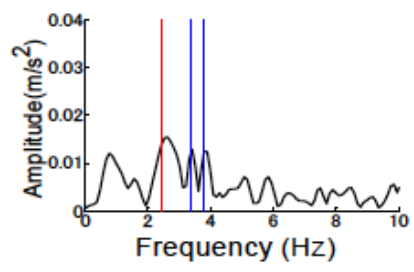

(a) FFT[Acc1]

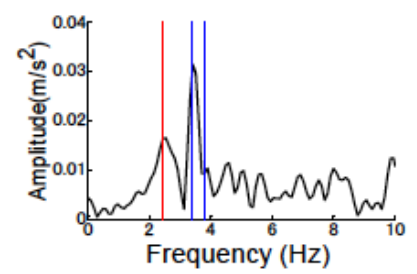

(c) FFT[Acc1-Acc2] Figure 6. Fourier spectra of trailer vibrations from laboratory experiment.

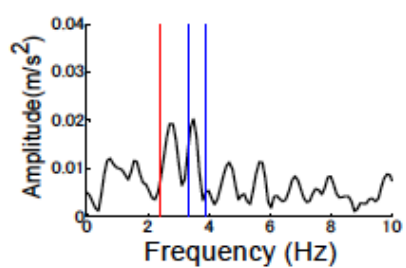

(b) FFT[Acc2]

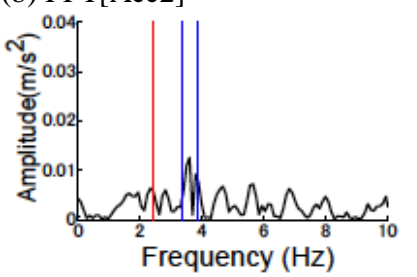

(d) FFT[Acc1]-FFT[Acc2] 
Table 4 Damage scenarios.

\begin{tabular}{|c|c|c|c|c|c|c|}
\hline \multirow{2}{*}{ Scenario } & \multirow{2}{*}{$\begin{array}{c}\text { mass } \\
(\mathrm{kg})\end{array}$} & \multicolumn{2}{|c|}{$f_{b 1}(\mathrm{~Hz})$} & \multicolumn{2}{|c|}{$f_{b 1-v} /(2 L) ; f_{b 1}+v /(2 L)$} & \multirow{2}{*}{$\begin{array}{c}\text { Damping } \\
\text { ratio } \\
\text { (Exp) }\end{array}$} \\
\hline & & Sim & Exp & Sim & Exp & \\
\hline Intact & 0 & 3.47 & 3.64 & $3.38 ; 3.57$ & $3.55 ; 3.74$ & 0.011 \\
\hline Damage1 & 8.3 & 3.37 & 3.50 & $3.28 ; 3.47$ & $3.41 ; 3.60$ & 0.009 \\
\hline Damage2 & 12.8 & 3.33 & 3.47 & $3.24 ; 3.43$ & $3.38 ; 3.57$ & 0.008 \\
\hline Damage3 & 17.2 & 3.29 & 3.42 & $3.20 ; 3.34$ & 3.33; 3.52 & 0.007 \\
\hline
\end{tabular}

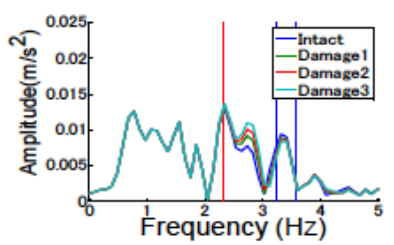

(a) FFT[Acc1]

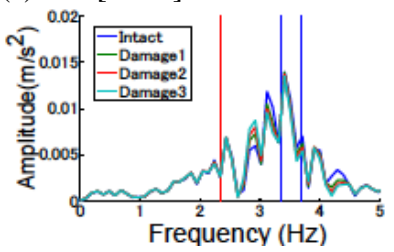

(c) FFT[Acc1-Acc2]

Figure 7. Fourier spectra of trailer vibrations from simulation w.r.t damage severity.

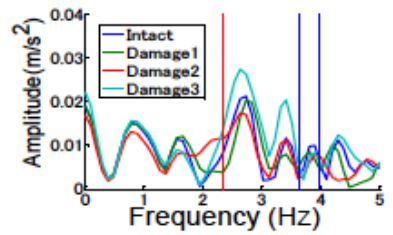

(a) FFT[Acc1]

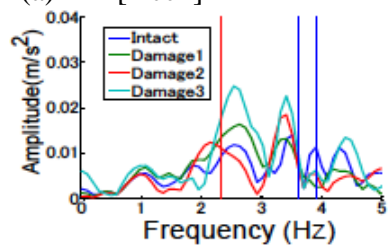

(c) FFT[Acc1-Acc2]

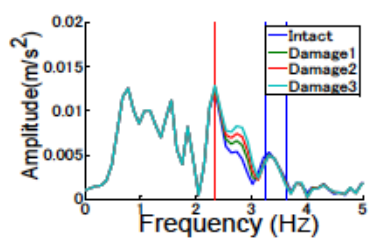

(b) FFT[Acc2]

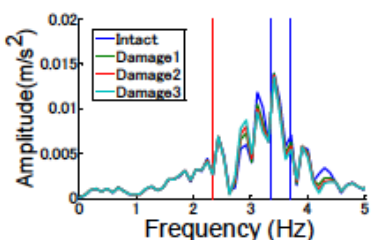

(d) FFT[Acc1]-FFT[Acc2]
Figure 8. Averaged Fourier spectra of trailer vibrations from laboratory experiments w.r.t damage severity.

On the other hand, in Figures 6(c) and 6(d), the peaks related to surface roughness and trailer frequency become smaller and the peaks close to the bridge natural frequency become dominant. It is noteworthy that the frequency close to the shifted bridge natural frequency $(3.55 \mathrm{~Hz})$ was also dominant as shown in Figure 6(c).

\section{FAULT DETECTION}

\subsection{Overview of fault detection}

The dynamic characteristics of the bridge may be changed when the bridge is damaged. In this study, to model pseudo damage, mass blocks were attached beneath the mid-span of the bridge. Four scenarios were investigated: Intact, Damage1, Damage2 and Damage3, each corresponding to $0 \mathrm{~kg}, 8.3 \mathrm{~kg}, 12.8$ $\mathrm{kg}$ and $17.2 \mathrm{~kg}$ attached mass respectively. The $1 \mathrm{st}$ natural frequency and the damping ratio of the bridge for each scenario are summarized in Table 4 .

\subsection{Results of fault detection}

Figure 7 shows the results of the numerical simulation for the fault detection. Fourier spectra of Acc1 and Acc2 are shown in Figures 7(a) and 7(b) respectively. Figure 7(c) shows FFT[Acc1-Acc2] and Figure 7(d) shows FFT[Acc1]-FFT[Acc2]. Again, vertical red line and blue lines in Figure 7 indicates the trailer frequency and the shifted bridge frequencies of the intact bridge, respectively. In Figures 7(a) and 7(b), the shift of bridge frequency-related peaks due to additional mass cannot be observed. However, the larger the additional mass is, the larger the Fourier amplitude in $2.73 \mathrm{~Hz}$ is.

Figure 8 shows Fourier spectra from the laboratory experiment for the fault detection. Figure 8 shows the averaged Fourier spectra of 10 runs of the measurement, from which it is difficult to identify changes in bridge natural frequencies. Despite of the difficulty in identifying bridge natural frequencies from vehicle vibrations, it is observed that the larger the additional mass is, the larger the peaks close to bridge frequencies become, as having been observed in the numerical simulation.

\subsection{Fault detection using MTS}

The Mahalanobis Taguchi System (MTS) is one of pattern recognition methods, which can be used in diagnostic applications to make quantitative decisions by constructing the multivariate measurement scale, Mahalanobis distance (MD) (Taguchi \& Jugulum 2000). In this study, MTS is applied in order to quantify the change in the statistical pattern of frequency distributions in Fourier spectra.

Figure 9 shows MDs of areas of Fourier spectra between a frequency band from the simulation for FFT[Acc1-Acc2] and FFT[Acc1]-FFT[Acc2], taking the amplitudes in the frequency band of $2.5 \mathrm{~Hz}$ to $3.0 \mathrm{~Hz}$ and $3.0 \mathrm{~Hz}$ to $3.5 \mathrm{~Hz}$ as variables respectively. In the simulation, 10 runs of moving test were performed for each scenario; each run was introduced a set of random noise of signal-to-noise ratio $(\mathrm{SNR})=$ 10. Observations from Intact case are taken as reference group for the other cases and a cross-validation (Bold 1982) was conducted for the same case. From Figure 9, changes in MDs from $2.5 \mathrm{~Hz}$ to $3.0 \mathrm{~Hz}$ anf from $3.0 \mathrm{~Hz}$ to $3.5 \mathrm{~Hz}$ could be observed with the increasing attached mass. This can be contributed to the shifted frequency of the bridge.

Figure 10 shows the MDs estimated from laboratory experiments for FFT[Acc1-Acc2] and FFT[Acc1]-FFT[Acc2], taking the amplitudes in the frequency range of $3.0 \mathrm{~Hz}$ to $3.5 \mathrm{~Hz}$ and $3.5 \mathrm{~Hz}$ to $4.0 \mathrm{~Hz}$ as variables respectively. 20 runs of the test for Intact case and 10 runs for Damage1, Damage2 and Damage3 were conducted. It is observed that MDs of FFT[Acc1]-FFT[Acc2] in $3.0 \mathrm{~Hz}$ to $3.5 \mathrm{~Hz}$ showed noticeable changes between the test scenarios. As for MDs of FFT[Acc1-Acc2], MDs of dam- 
age cases were larger than MDs of Intact case. Changes in MDs considering from $3.5 \mathrm{~Hz}$ to $4.0 \mathrm{~Hz}$, which covers the shifted frequency of the damaged bridge, were also observed.
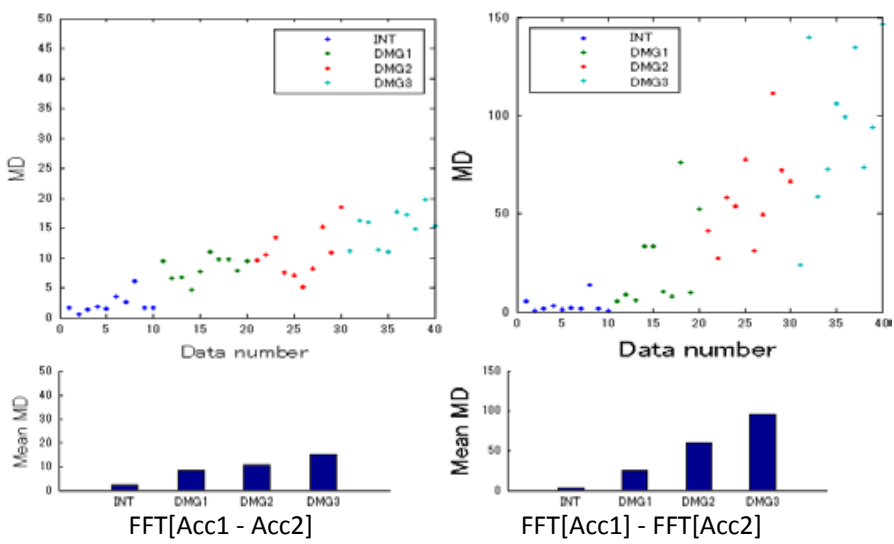

(a) $2.5 \mathrm{~Hz}$ to $3.0 \mathrm{~Hz}$
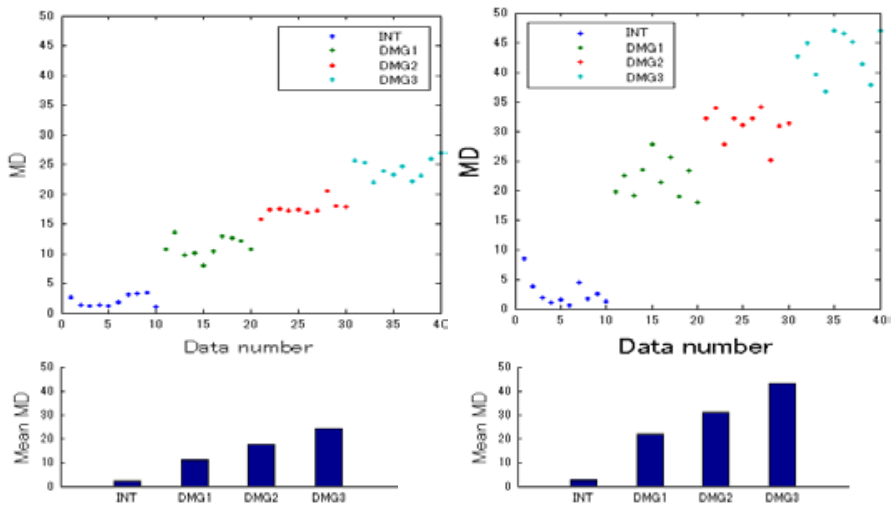

FFT[Acc1 - Acc2]

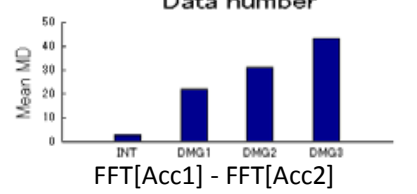

(b) $3.0 \mathrm{~Hz}$ to $3.5 \mathrm{~Hz}$

Figure 9. MDs of areas of Fourier spectra (numerical simulation).
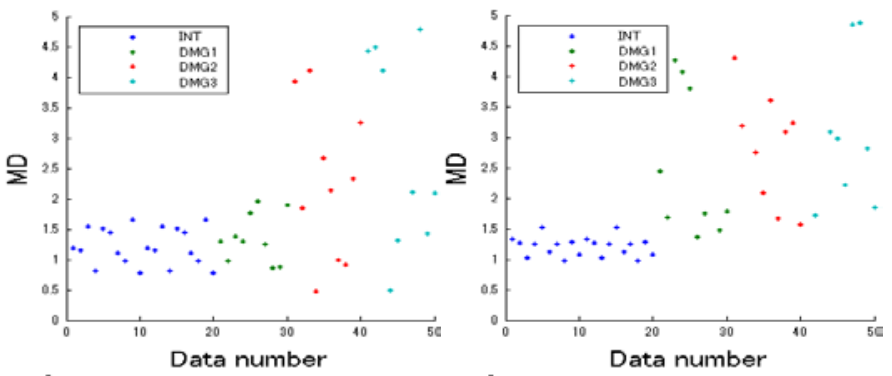

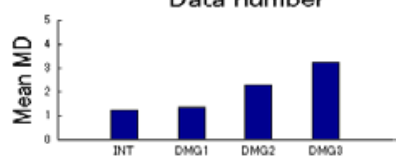

FFT[Acc1 - Acc2]

(a) $3.0 \mathrm{~Hz}$ to $3.5 \mathrm{~Hz}$
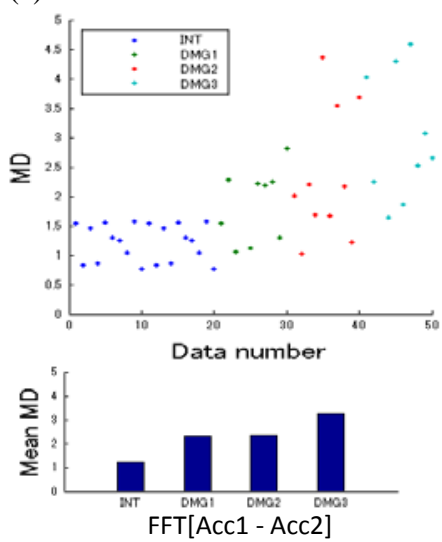

(b) $3.5 \mathrm{~Hz}$ to $4.0 \mathrm{~Hz}$

Figure 10. MDs of areas of Fourier spectra (laboratory experiment).

\section{CONCLUDING REMARKS}

A drive-by inspection approach using two passing trailers was investigated through a theoretical study, a numerical simulation and laboratory experiment. Observations are summarized as follows.

1) By subtracting the synchronized responses of the two vehicles from each other, it can be possible to eliminate the effect of road surface roughness theoretically.

2) The numerical simulation verified the effectiveness of subtracting the synchronized responses from two trailers to reduce the effect of road surface roughness.

3) In the laboratory experiment, subtracting the synchronized responses from two trailers resulted in reduction of the effect of road surface roughness, i.e. the amplitudes of the frequency lower than $2 \mathrm{~Hz}$ were reduced.

4) For fault detection, in both the numerical simulation and laboratory experiment, the amplitude of frequency near the shifted bridge frequencies increased with the increasing damage level.

5) Taking the observations from the intact case as reference and performing MTS to the damage cases, MDs of areas of Fourier spectra between the shifted bridge frequencies increased as damage level increased, and demonstrated feasibility of the fault detection by the drive-by monitoring.

6) FFT[Acc1]-FFT[Acc2] was recommended in practical uses considering difficulties to synchronize Acc1 and Acc2 in the time domain.

\section{REFERENCES}

Bold, B. 1982. Famous problems of geometry and how to solve them. New York, Dover.

Kim, CW, Isemoto, R, McGetrick, PJ, Kawatani, M, and O'Brien, EJ. 2014. Drive-by bridge inspection from three different approaches, Smart Structures and Systems, 13(5), 775-796.

Lin, CW, Yang, YB. 2005. Use of a passing vehicle to scan the fundamental bridge frequencies: An experimental verification, Engineering Structures 27, 1845-1878.

McGetrick, PJ, Gonzalez, A and Obrien EJ. 2009. Theoretical investigation of the use of a moving vehicle to identify bridge dynamic parameters, Insight 51(8), 433-438.

Taguchi, G and Jugulum, R. 2000. New trends in multivariate diagnosis, Indian Journal of Statistics, Vol. 62, Series B, pp. 233-248.

Toshinami, T, Kawatani, M and Kim, CW. 2010. Feasibility investigation for identifying bridge's fundamental frequencies from vehicle vibrations, Proc. of The $5^{\text {th }}$ International Conference on Bridge Maintenance, safety and Management, July 11-15 Philadelphia, Pennsylvania, USA.

Yang, YB, Lin, CW and Yau, JD. 2004. Extracting bridge frequencies from the dynamic response of a passing vehicle, Journal of Sound and Vibration 272, 471-493.

Yau, JD, Yang, YB, Kuo, SR. 1999. Impact response of high speed rail bridges and riding comfort of rail cars, Engineering Structures 21, 836-844.

Yang, YB, Li, YC, and Chang, KC. 2012. Effect of road surface roughness on the response of a moving vehicle for identification of bridge frequencies, Interaction and Multiscale Mechanics 5(4), 347-368. 